\title{
Training, training and more training
}

\author{
Rosa Sicari ${ }^{1}$
}

Published online: 17 May 2021

(c) The Author(s), under exclusive licence to Springer Nature B.V. 2021

The non-ischemic applications of stress echocardiography are still restricted to few research laboratories that are able to display a wide clinical case mix scenarios and highly skilled operators. Operators profile with highly skilled expertise is the mainstay of stress echo quality, it is also a reason for its limited application outside well beaten pathways. With the RIGHT heart international NETwork (RIGHT-NET), Ferrara et al. [1] demonstrate beyond any reasonable doubt that an appropriate training, with strict criteria allows a very high feasibility of stress echo applied to the right heart. The trial assesses a large sample of consecutive subjects, collected in a 6 months period of time and showing a very high feasibility in assessing right heart echo parameters during semisupine stress. Technique, parameters, clinical case scenarios show that not only we should do it but, most important, that we can do it. No data on the clinical implications and results are reported because the trial was focused only on feasibility, reminding us all that sometimes, in clinical research we have to re-start from the basics. Authors should be definitively congratulated for the effort and for their ability to share their view on how to perform right heart stress echocardiography. Overall, feasibility is very high but not all parameters are created equal and some are less performing than others even in the hands of high skilled centers. The bottom-line is that, of the wealth of information that we may obtain we must stick to the simplest ones. Again, if we can make it we have to make it simple. Otherwise right heart stress echo will never be a clinical player in the real world. The second message that we read in transparency out of these results is that training never stops in stress echocardiography and the more we do it the more we should learn how to do it. Training is the key word and learned personnel keep learning from the way they shape the exam on the basis of a tailored approach to clinical non-invasive diagnosis. Authors did not use strain, more suited to less experienced operators. It is probably another philosophical choice in order to improve feasibility in large samples of consecutive patients. Moreover, one of the strengths of right heart stress echocardiography is that its parameters are quantitative. And this goes back to the other main limitation of stress echocardiography: impressionistic or too operator-dependency in interpretation is a drawback of the technique that need to be solved. Numbers, quantity albeit imperfect (Authors acknowledge the fact that none of the non-invasive parameters were compared to invasive studies.) is what is necessary in stress echocardiography. Right heart stress echocardiography providing pressures, functions in numbers seem to be the appropriate candidate to screen patients with clinical conditions ranging from healthy subjects to patients at risk or/with overt PH. What's next? Human intelligence works better when knowledge and learning is continuous. This study is giving us a very simple but unavoidable message in medicine, train, train better and train again.

\section{Reference}

1. Ferrara F et al (2021) Feasibility of semi-recumbent bicycle exercise Doppler echocardiography for the evaluation of the right heart and pulmonary circulation unit in different clinical conditions: the RIGHT heart international NETwork (RIGHT-NET). Int J Cardiovasc Imaging. https://doi.org/10.1007/s10554-021-02243-X

Publisher's Note Springer Nature remains neutral with regard to jurisdictional claims in published maps and institutional affiliations.
Rosa Sicari

rosas@ifc.cnr.it

1 Institute of Clinical Physiology, CNR, Via G. Moruzzi, 1, 56124 Pisa, Italy 